\title{
Ubiquity, Diversity, and Genomic Complexity of Cyanophages in Freshwater Environments ${ }^{+}$
}

\author{
Qi-Ya Zhang ${ }^{1,2}$ \\ 1 State Key Laboratory of Freshwater Ecology and Biotechnology, Institute of Hydrobiology, \\ Chinese Academy of Sciences, Wuhan 430072, China; zhangqy@ihb.ac.cn \\ 2 The Innovation Academy of Seed Design, Chinese Academy of Sciences, Beijing 100101, China \\ † Presented at Viruses 2020-Novel Concepts in Virology, Barcelona, Spain, 5-7 February 2020. \\ Published: 24 June 2020
}

\begin{abstract}
Cyanophages are viruses that infect cyanobacteria (also known as blue-green algae) and are ubiquitious in marine and freshwater environments. In recent years, freshwater cyanophages have attracted more attention because they can affect global freshwater ecosystems. The spatial distribution and morphological diversity of cyanophage populations were examined in Lake Donghu with three trophic regions: hypertrophic, eutrophic, and mesotrophic regions. The surprisingly high viral abundance (ranging from $10^{8}$ to $10^{9}$ phage $\mathrm{mL}^{-1}$ ) and morphological diversity were detected. Most of them have tails and belong to the families Siphoviridae, Myoviridae, and Podoviridae. Various morphotypes were observed, such as prolate-headed virus-like particles and lemon-shaped virus-like particles. In addition, some cyanophages were studied by virological experiments and whole-genome analyses, combined with morphological observation. For example, three cyanophages were isolated and their whole genomes were sequenced. Contractile tail myonophage MaMV-DC infects bloom-forming cyanobacterium Microcystis aeruginosa. Tailless cyanophage Planktothrix agardhii virus isolated from Lake Donghu (PaV-LD) infects filamentous cyanobacterium. Short-tail podovirus A- $4 \mathrm{~L}$ can infect the model cyanobacterium Anabaena sp. strain PCC 7120. The MaMV-DC genome contains 169,223 bp encoding 170 putative open reading frames (ORFs). The PaV-LD genome posseses 95,299 bp encoding 142 putative ORFs. The genome of shorttail podovirus A-4L has 41,750 bp encoding 38 putative ORFs. There are significant differences in their genomic size and encoded tail proteins, but all three cyanophages contain genes that are not commonly found in phages. By studying the vast biodiversity of viruses in freshwater environments, these novel findings of cyanophages broaden our insights, and allow us to gain more useful knowledge about the global impact of these viruses in freshwater ecosystems.
\end{abstract}

Keywords: cyanophages; genomic complexity; freshwater environment

Funding: This research was supported by grants from the National Key R\&D Programmes of China (2018YFA0903101).

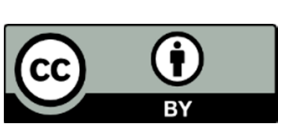

(C) 2020 by the authors. Licensee MDPI, Basel, Switzerland. This article is an open access article distributed under the terms and conditions of the Creative Commons Attribution (CC BY) license (http://creativecommons.org/licenses/by/4.0/). 